\title{
Construction of fast xylose-fermenting yeast based on industrial ethanol-producing diploid Saccharomyces cerevisiae by rational design and adaptive evolution
}

\author{
Liuyang Diao ${ }^{1,2}$, Yingmiao Liu ${ }^{1,2}$, Fenghui Qian ${ }^{1,2}$, Junjie Yang ${ }^{1,2}$, Yu Jiang ${ }^{1,2}$ and Sheng Yang ${ }^{1,2^{*}}$
}

\begin{abstract}
Background: It remains a challenge for recombinant S. cerevisiae to convert xylose in lignocellulosic biomass hydrolysates to ethanol. Although industrial diploid strains are more robust compared to laboratory haploid strains, however, industrial diploid S. cerevisiae strains have been less pursued in previous studies. This work aims to construct fast xylose-fermenting yeast using an industrial ethanol-producing diploid S. cerevisiae strain as a host.

Results: Fast xylose-fermenting yeast was constructed by genome integration of xylose-utilizing genes and adaptive evolution, including 1) Piromyces XYLA was introduced to enable the host strain to convert xylose to xylulose; 2) endogenous genes (XKS1, RKI1, RPE1, TKL1, and TAL1) were overexpressed to accelerate conversion of xylulose to ethanol; 3) Candida intermedia GXF1, which encodes a xylose transporter, was introduced at the GRE3 locus to improve xylose uptake; 4) aerobic evolution in rich xylose media was carried out to increase growth and xylose consumption rates. The best evolved strain CIBTS0735 consumed $80 \mathrm{~g} / \mathrm{l}$ glucose and $40 \mathrm{~g} / \mathrm{l}$ xylose in rich media within 24 hours at an initial $\mathrm{OD}_{600}$ of 1.0 (0.63 g DCW/I) and produced $53 \mathrm{~g} / \mathrm{l}$ ethanol.
\end{abstract}

Conclusions: Based on the above fermentation performance, we conclude that CIBTS0735 shows great potential for ethanol production from lignocellulosic biomass.

Keywords: Saccharomyces cerevisiae, Xylose isomerase, Adaptive evolution, Xylose fermentation

\section{Background}

As a transportation fuel, ethanol has the potential to displace a substantial portion of gasoline. In recent years, ethanol production from lignocellulosic biomass has been attracting great attention. To produce ethanol, raw materials such as corn stover should be first pretreated and then hydrolyzed to liberate simple sugars, i.e. glucose and xylose. Subsequently, these sugars are converted to ethanol by microbial fermentation [1]. Saccharomyces cerevisiae is believed to be the most promising biocatalyst for this conversion due to its wide use in the starch- and

\footnotetext{
* Correspondence: syang@sibs.ac.cn

${ }^{1}$ CAS Key Laboratory of Synthetic Biology, Institute of Plant Physiology and Ecology, Shanghai Institutes for Biological Sciences, Chinese Academy of Sciences, 300 Fenglin Road, Shanghai 200032, China

${ }^{2}$ Shanghai Research and Development Center of Industrial Biotechnology, 528 Ruiqing Road, Shanghai 201201, China
}

sucrose-based ethanol industry [2]. However, S. cerevisiae cannot ferment xylose into ethanol. Since xylose is the second most abundant sugar present in the biomass hydrolysate after glucose, therefore fast xylose fermentation is required to produce ethanol from lignocellulosic biomass economically [3].

Great progress has been achieved to make $S$. cerevisiae able to ferment xylose in the last decade [4-8]. S. cerevisiae can take up xylose by nonspecific transporters [9]. After entering into cells, xylose can be converted to xylulose by either the oxidoreductive pathway or the isomerization pathway [2]. Since both pathways are absent in S. cerevisiae, therefore heterologous enzymes have to be introduced. Compared to the oxidoreductive pathway, the isomerization pathway receives more attention since it does not

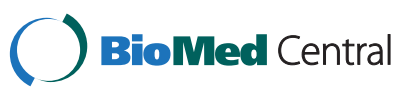

(c) 2013 Diao et al.; licensee BioMed Central Ltd. This is an open access article distributed under the terms of the Creative Commons Attribution License (http://creativecommons.org/licenses/by/2.0), which permits unrestricted use, distribution, and reproduction in any medium, provided the original work is properly cited. 
have a cofactor imbalance issue [8]. After phosphorylation by endogenous xylulokinase, xylulose enters the pentose phosphate pathway and then glycolysis to produce ethanol [10]. In previous studies, several steps have been identified as bottlenecks that limit the xylose consumption rate, including slow xylose uptake [11], slow conversion of xylose to xylulose [8], and limited flux of the pentose phosphate pathway [12]. Overexpression of xylose-utilizing proteins, to some extent, can remove these bottlenecks $[5,11]$. Moreover, GRE3 is thought to cause xylitol accumulation and its deletion is beneficial to minimize xylitol formation [13]. As well as genetic manipulations, adaptive evolution is necessary to increase the xylose consumption rate. For example, several studies showed that combinatorial use of genetic manipulations (i.e. introduction of $X Y L A$ from Piromyces and overexpression of endogenous XKS1, RPE1, RKI1, TAL1, and TKL1) and adaptive evolution in xylose media can generate efficient xylose-utilizing strains $[4,8,14]$. However, there are two drawbacks for these studies: 1) laboratory haploid strains were chosen as hosts, which are generally considered not as robust as industrial diploid strains when fermenting lignocellulosic biomass hydrolysates [1] and 2) plasmid-based protein expression was employed, which is regarded as not stable as integration-based protein expression $[15,16]$. Although genome integration of xylose isomerase was pursued by Tanino et al [17], however, a laboratory haploid strain was selected to construct xylose-fermenting yeast. Until recently, a xylose-fermenting $S$. cerevisiae strain was reported based on an industrial diploid strain and genome integration of xylose isomerase and other genes [18].

So far, fermenting xylose in lignocellulosic biomass hydrolysates remains a challenge. Although industrial diploid strains are more robust compared to laboratory haploid strains, however, industrial diploid S. cerevisiae strains have been less pursued in previous studies. This study aims to construct fast xylose-fermenting yeast using an industrial ethanol-producing diploid S. cerevisiae strain as a host. For this purpose, S. cerevisiae CCTCC M94055 was chosen as the host. This strain is widely used to produce starch-based fuel and drinking ethanol and possesses phenotypes desired for industrial use, such as high tolerance to high temperatures, low $\mathrm{pH}$ value, and high ethanol and inhibitor concentrations [19,20]. To avoid unstable plasmid-based protein expression, we integrated all genes into chromosomes by homologous recombination. Specifically, in addition to introduction of XYLA and overexpression of XKS1, RPE1, RKI1, TAL1, TKL1, we also introduced a xylose transporter-encoding gene GXF1 from Candida intermedia at the GRE3 locus. After simple aerobic evolution in rich xylose media, the best evolved strain CIBTS0735 consumed $80 \mathrm{~g} / \mathrm{l}$ glucose and $40 \mathrm{~g} / \mathrm{l}$ xylose in 24 hours at an initial $\mathrm{OD}_{600}$ of $1.0(0.63 \mathrm{~g} \mathrm{DCW} / \mathrm{l})$ and produced $53 \mathrm{~g} / \mathrm{l}$ ethanol.

\section{Results}

Rational construction of xylose-fermenting S. cerevisiae

Figure 1 shows the whole process for strain construction. To convert xylose to xylulose in cells, two copies of a eukaryotic xylose isomerase-encoding gene Piromyces $X L Y A$ were integrated at the ARG1 and Ty1 loci sequentially. A strong promoter TPI1p was used to drive XLYA expression. Then, an additional copy of genes encoding xylulokinase $(X K S 1)$ and four non-oxidative enzymes of the pentose phosphate pathway (PPP, RKI1, RPE1, TKL1, and TAL1) were inserted at the $\delta$ locus, resulting in CIBTS0525. These genes were all equipped with strong promoters to achieve high expression and to accelerate conversion of xylulose to ethanol. Subsequently, a xylose transporter-encoding gene GXF1 was integrated at the GRE3 locus to increase the xylose uptake rate and the resultant strain was CIBTS0573. This integration inactivated one of the two copies of GRE3.

\section{Adaptive evolution to obtain fast xylose-fermenting S. cerevisiae}

Although CIBTS0525 and CIBTS0573 contained a xylosefermenting pathway, however both strains grew and consumed xylose slowly even under aerobic conditions (Figure 2 and Table 1). To select for spontaneous mutants with improved growth and xylose consumption rates, both strains were subjected to serial transfer in YP medium supplemented with xylose under aerobic conditions. After ca. 10 transfers, the growth and xylose consumption rates of both strains started to increase. Then additional several transfers were carried out for both strains until no improvement was observed. From the final transfers,

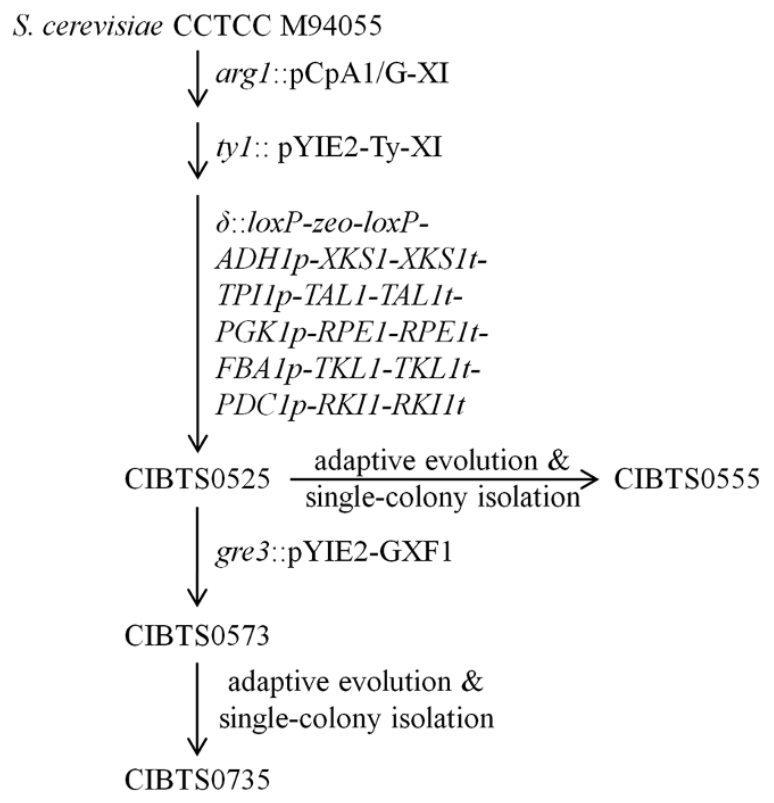

Figure 1 Schematic diagram of strain construction. 

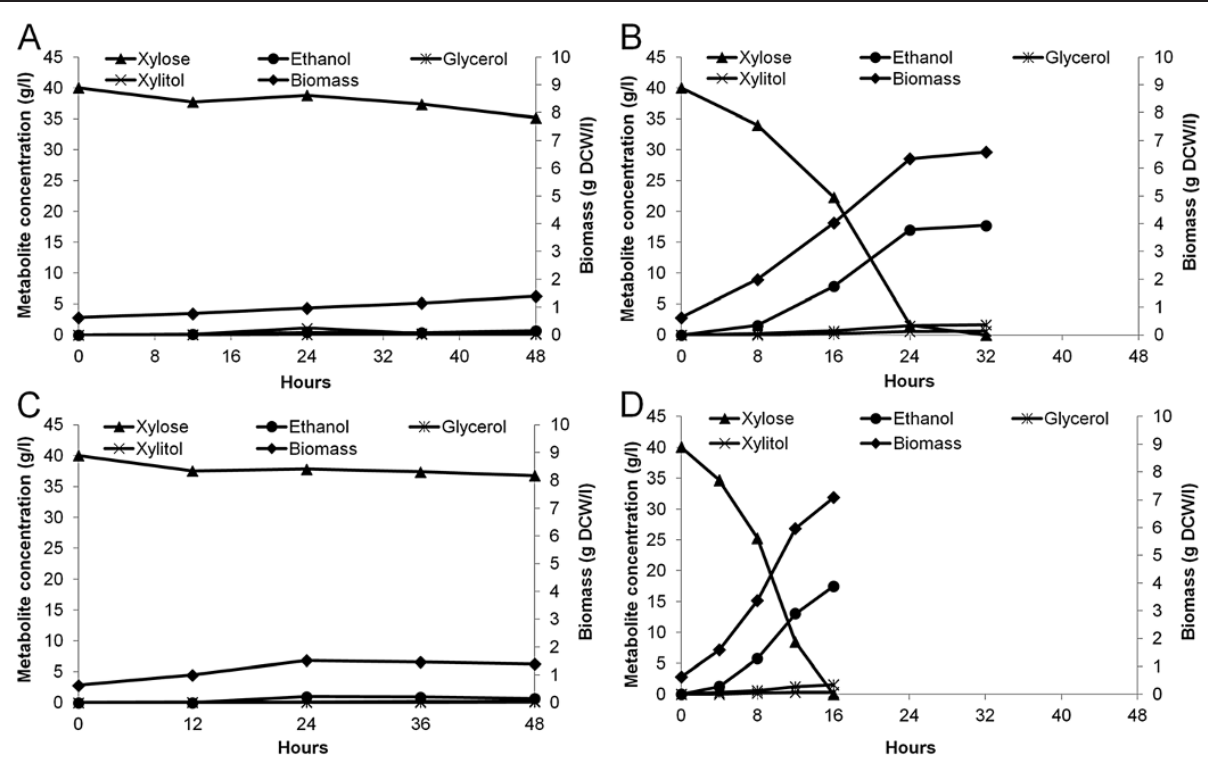

Figure 2 Anaerobic fermentation of strains on xylose. (A), CIBTS0525; (B), CIBTS0555; (C), CIBTS0573; (D), CIBTS0735. Strains were cultured

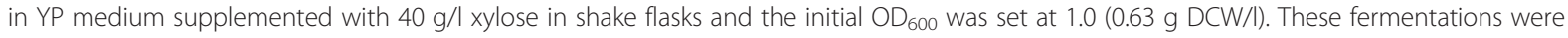
performed only once.

both cultures were diluted and plated on YPX agar plates. Multiple colonies of each strain were inoculated into YP medium supplemented with $40 \mathrm{~g} / \mathrm{l}$ xylose to examine their xylose consumption rates. The two best strains CIBTS0555 and CIBTS0735 were obtained from CIBTS 0525 and CIBTS0573 respectively. As previously reported $[8,14]$, these data show that adaptive evolution is required to obtain fast xylose-fermenting S. cerevisiae as well as metabolic engineering.

Next, the two evolved strains CIBTS0555 and CIBTS 0735 were characterized with respect to their growth and xylose fermentation rates in YP medium supplemented with $40 \mathrm{~g} / \mathrm{l}$ xylose, using their unevolved parents as controls (Figure 2 and Table 1). For both the unevolved strains CIBTS0525 and CIBTS0573, cells grew very slowly with growth rates below $0.02 \mathrm{~h}^{-1}$ and also consumed xylose very slowly (Figure 2A, Figure 2C and Table 1 ).
After adaptive evolution, in strikingly contrast, CIBTS0555 showed a 6-fold increase of the growth rate and its xylose consumption rate was increased to $0.505 \mathrm{~g} / \mathrm{g} \mathrm{DCW} / \mathrm{h}$ (Figure 2B and Table 1). CIBTS0735 even displayed a 10fold increase of the growth rate and its xylose consumption rate reached $0.957 \mathrm{~g} / \mathrm{g} \mathrm{DCW} / \mathrm{h}$ (Figure 2D and Table 1). For both strains CIBTS0555 and CIBTS0735, ethanol yields exceeded $0.41 \mathrm{~g} / \mathrm{g}$ xylose, while glycerol yields were kept low (below $0.04 \mathrm{~g} / \mathrm{g}$, Table 1) and no xylitol accumulation was detected. Taken together, these data clearly show that adaptive evolution significantly improves xylose fermentation and ethanol production.

\section{GXF1 expression might contribute to increase growth and xylose consumption rates}

A previous study showed that xylose uptake is a bottleneck for xylose fermentation and therefore, expression of

Table 1 Fermentation performance of xylose-fermenting S. cerevisiae strains

\begin{tabular}{|c|c|c|c|c|c|c|}
\hline Strain & CCTCC M94055 & CIBTS0525 & CIBTS0555 & CIBTS0573 & CIBTS0735 & CIBTS0735 \\
\hline Medium $^{a}$ & YPX40 & YPX40 & YPX40 & YPX40 & YPX40 & YPD80X40 \\
\hline Specific growth rate ${ }^{b}, h^{-1}$ & N/A & 0.016 & 0.096 & 0.019 & 0.187 & 0.181 \\
\hline Ethanol yield ${ }^{c}, \mathrm{~g} / \mathrm{g}$ & N/A & N/A & 0.443 & N/A & 0.412 & 0.454 \\
\hline Glycerol yield ${ }^{c}, g / g$ & N/A & N/A & 0.039 & N/A & 0.039 & 0.041 \\
\hline Xylitol yield ${ }^{c}, \mathrm{~g} / \mathrm{g}$ & N/A & N/A & N/A & N/A & N/A & N/A \\
\hline Sugar consumption rate ${ }^{c}, g / g$ DCW/h & N/A & N/A & 0.505 & N/A & 0.957 & 1.300 \\
\hline Ethanol production rate ${ }^{c}, \mathrm{~g} / \mathrm{g}$ DCW/h & N/A & N/A & 0.224 & N/A & 0.394 & 0.590 \\
\hline
\end{tabular}

${ }^{\mathrm{a}}$ YPX40, YP medium supplemented with $40 \mathrm{~g} / \mathrm{l}$ xylose; YPD80X40, YP medium supplemented with $80 \mathrm{~g} / \mathrm{l}$ glucose and $40 \mathrm{~g} / \mathrm{l}$ xylose. ${ }^{\mathrm{b}} \mathrm{Specific}$ growth rates were calculated from the exponential growth phase. 'Xylose consumption and ethanol production rates were calculated as described previously [21]. These two parameters as well as ethanol and xylitol yields were calculated from the growth phase (Figures 2 and 3, from the beginning of fermentation to below $10 \mathrm{~g} / \mathrm{l}$ residual xylose). 
GXF1 encoding a xylose transporter can accelerate xylose utilization [11]. In this study GXF1 also was introduced into CIBTS0525 to further increase the xylose consumption rate, resulting in CIBTS0573. Like CIBTS0525, CIBTS0573 also grew very slowly with a growth rate of $0.019 \mathrm{~h}^{-1}$ in YP medium supplemented with $40 \mathrm{~g} / \mathrm{l}$ xylose. After adaptive evolution, compared to CIBTS0555 (evolved from CIBTS0525), CIBTS0735 (evolved from CIBTS0573) showed a 95\% increase of the growth rate and a $90 \%$ increase of the xylose consumption rate (Figure 2D versus Figure 2B and Table 1). Accordingly, the ethanol production rate of CIBTS0735 was increased to $0.394 \mathrm{~g} / \mathrm{g} \mathrm{DCW} / \mathrm{h}$, which is $76 \%$ higher than that of CIBTS0555 (Figure 2D vs Figure 2B and Table 1). Besides, prolonged adaptive evolution of CIBTS0555 did not generate a strain displaying better growth and fermentation performance. These results imply that GXF1 expression might contribute to increase growth and xylose consumption rates. Moreover, since CIBTS0555 and CIBTS0735 were evolved independently, we cannot exclude the possibility that different mutations might have occurred in the two evolved strains.

\section{Mixed sugars can be cofermented efficiently into ethanol}

To test whether mixed sugars can be cofermented efficiently, the best evolved strain CIBTS0735 was characterized in YP medium supplemented with $80 \mathrm{~g} / \mathrm{l}$ glucose and $40 \mathrm{~g} / \mathrm{l}$ xylose. As shown in Figure 3, both sugars were consumed in $24 \mathrm{~h}$ with an ethanol yield of $0.45 \mathrm{~g} / \mathrm{g}$ sugar. The total sugar consumption rate was $1.3 \mathrm{~g} / \mathrm{g}$ $\mathrm{DCW} / \mathrm{h}$, which is $36 \%$ higher than that of CIBTS0735 cultivated in YP medium supplemented with $40 \mathrm{~g} / \mathrm{l}$ xylose. However, although both sugars were utilized in $24 \mathrm{~h}$, xylose was not significantly consumed until glucose was depleted. As for byproducts, glycerol yields were also kept low $(0.041 \mathrm{~g} / \mathrm{g}$, Table 1$)$ and no xylitol accumulation was detected. Taken together, although glucose and xylose

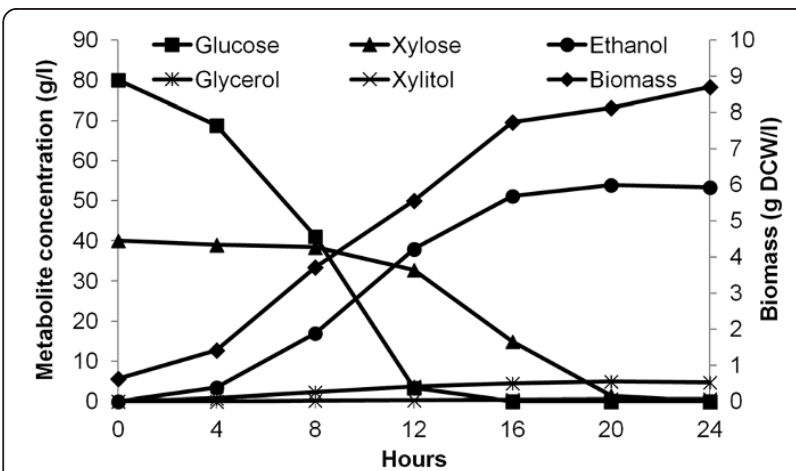

Figure 3 Anaerobic fermentation of CIBTS0735 on mixed sugars. CIBTS0735 was cultured in YP medium supplemented with $80 \mathrm{~g} / \mathrm{l}$ glucose and $40 \mathrm{~g} / \mathrm{l}$ xylose in a shake flask and the initial $\mathrm{OD}_{600}$ was set at $1.0(0.63 \mathrm{~g} \mathrm{DCW/I)}$. This fermentation was performed only once. were consumed sequentially, these data clearly show that mixed sugars can be cofermented efficiently into ethanol.

\section{CIBTS0735 exhibits a higher activity of xylose isomerase}

A recent study showed that an increased activity of xylose isomerase was observed during adaptive evolution, which in part contributed to efficient xylose assimilation [8]. In this study, we also examined whether the activity of xylose isomerase had increased during the adaptive evolution of CIBTS0735. To do this, xylose isomerase activities of strains CIBTS0573 and CIBTS0735 were compared. As shown in Figure 4, after adaptive evolution, a $100 \%$ increase of the xylose isomerase activity was observed for CIBTS0735. This result suggests that the elevated activity of xylose isomerase might have contributed to the efficient xylose fermentation of CIBTS0735.

\section{Discussion}

Fast xylose fermentation is a key technology to produce ethanol from lignocellulosic biomass economically. Although great progress has been achieved in the last decade, however xylose fermentation remains a challenge today. Based on an industrial ethanol-producing diploid strain S. cerevisiae CCTCC M94055, we constructed fast xylose-fermenting $S$. cerevisiae by genome integration of xylose-utilizing genes in this study.

Strong promoter-driven expression of $X Y L A, X K S 1$, and four genes of the non-oxidative PPP did not immediately result in obvious growth on xylose. For example, aerobic growth rates of CIBTS0525 and CIBTS0573 were $0.016 \mathrm{~h}^{-1}$ and $0.019 \mathrm{~h}^{-1}$, respectively. Although these two strains grew at rates comparable to similarly constructed strains H131-A3 (growth rate, $0.031 \mathrm{~h}^{-1}$ ) [8] and BSPC095 (growth rate, $0.009 \mathrm{~h}^{-1}$ ) [14], their growth rates were substantially lower than that of RWB217 (growth rate, $0.22 \mathrm{~h}^{-1}$ ) [5]. This might be caused by 1 ) only two copies of XYLA integrated into the genome and 2) a different

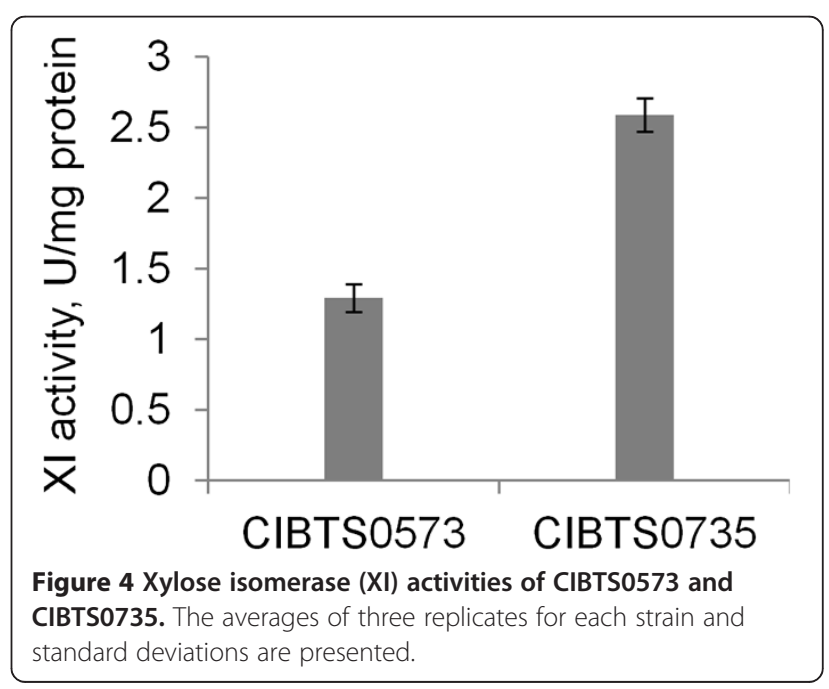


host strain used. By adapting CIBTS0525 on xylose, we obtained CIBTS0555 that showed a growth rate of $0.096 \mathrm{~h}^{-1}$ in rich xylose media. In parallel, we also engineered another industrial ethanol-producing diploid S. cerevisiae strain CICC 1300 using the same strategy as CCTCC M94055 (Additional file 1: Figure S1). The resultant strain CIBTS0552 displayed a comparable growth rate $\left(0.097 \mathrm{~h}^{-1}\right)$ to that of CIBTS0555, suggesting that the engineering strategy is applicable to not only laboratory strains $[5,8,14]$, but industrial strains.

Previous studies showed that GXF1 expression is beneficial to xylose fermentation in S. cerevisiae $[6,11,22,23]$ and these studies were all carried out using recombinant yeast strains with a limited flux of xylose metabolism. In this study, we show that GXF1 expression might contribute to increase growth and xylose consumption rates. This notion could be further supported by additional evidence. In parallel to GXF1, we also introduced into CIBTS0525 another xylose transporter-encoding gene SUT1 from Pichia stipitis (Additional file 1: Figure S2). After adaptive evolution, the resultant strain (CIBTS0734) exhibited a xylose consumption rate of $0.800 \mathrm{~g} / \mathrm{g} \mathrm{DCW} / \mathrm{h}$ in rich xylose media, which is $58 \%$ higher than that of CIBTS0555 $(0.505 \mathrm{~g} / \mathrm{g} \mathrm{DCW} / \mathrm{h})$. However, without introducing heterologous xylose transporters, no further improvement was observed for CIBTS0555 through prolonged adaptation on xylose. Besides, these results also suggest that xylose uptake is a bottleneck for CIBTS0555 that displays a relatively high flux of xylose consumption.

It has been reported that anaerobic xylose-limited continuous cultivation in a chemostat was required to reach maximal growth rates, as well as (an)aerobic sequential batch cultivation [6]. However, in this study only simple aerobic batch cultivation was employed to generate fast-growing CIBTS0735 with a growth rate of $0.187 \mathrm{~h}^{-1}$ in rich xylose media. The reason for dispensability of chemostat cultivation is probably attributed to GXF1 expression, since the purpose of xylose-limited chemostat cultivation is to improve xylose uptake $[4,8]$ which is the same as the purpose of GXF1 expression.

Table 2 compares several best-performing xylose-fermenting strains. From this overall comparison, we know that CIBTS0735 displays comparable or even superior phenotypes. However, it should be noted that the fermentation for these strains was carried out under different conditions. Moreover, CIBTS0735 was also examined in the undetoxified corn stover hydrolysate provided by National Renewable Energy Laboratory and Novozymes A/S. Although under harsh conditions (e.g. $8.4 \mathrm{~g} / \mathrm{l}$ acetic acid and $2.6 \mathrm{~g} / \mathrm{l} \mathrm{HMF),} \mathrm{CIBTS0735} \mathrm{still} \mathrm{converted} 82.3 \mathrm{~g} / \mathrm{l}$ glucose and $46.7 \mathrm{~g} / \mathrm{l}$ xylose to $51.8 \mathrm{~g} / \mathrm{l}$ ethanol (Additional file 1: Figure S3). This result suggests that CIBTS0735 is a potential biocatalyst for ethanol production from lignocellulosic biomass. However, compared to the xylose fermentation in the rich media, the xylose utilization in the corn stover hydrolysate was much slower, which was probably caused by the presence of high concentrations of inhibitors. Rational design and adaptation against inhibitors may be pursued to increase xylose fermentation in the undetoxified corn stover hydrolysate [24-28].

Evolutionary engineering is a powerful tool to generate strains with desired production traits [29]. Several studies employed this tool to generate improved xylose-fermenting yeast $[4,8,14,30]$. However, this technique provides no information on genetic changes. For CIBTS0735, the increased activity of xylose isomerase is likely to contribute to its fast growth and efficient xylose fermentation. Previous studies also observed an increase of the xylose isomerase activity after adaptive evolution $[8,14]$. Except for the elevated xylose isomerase activity, other mutations might also have occurred in CIBTS0735 to account for improved phenotypes, including increased xylose transport [4], balanced xylose metabolic flux [8], and even genome-scale changes $[8,14]$. However, accurate genetic changes occurred in CIBTS0735 remain to be elucidated by genome resequencing and transcriptome analysis and this work will be pursued in our following research.

\section{Conclusions}

Here we report the construction of fast xylose-fermenting yeast CIBTS0735 based on xylose isomerase and industrial diploid S. cerevisiae. This strain can convert $80 \mathrm{~g} / \mathrm{l}$ glucose and $40 \mathrm{~g} / \mathrm{l}$ xylose to $53 \mathrm{~g} / \mathrm{l}$ ethanol in 24 hours at initial $\mathrm{OD}_{600}$ of $1.0(0.63 \mathrm{~g} \mathrm{DCW} / \mathrm{l})$ and therefore shows great potential for fuel ethanol production from lignocellulosic biomass.

\section{Methods}

\section{Strains and plasmids}

Strains and plasmids used in this study are listed in Table 3. Escherichia coli DH5 $\alpha$ was used as a cloning host. S. cerevisiae CCTCC M94055 was used as the host strain to construct xylose-fermenting yeast.

\section{Media and culture conditions}

E. coli strains were grown in $\mathrm{LB}$ medium at $37^{\circ} \mathrm{C}$, $250 \mathrm{rpm}$ and when necessary, $100 \mu \mathrm{g} / \mathrm{ml}$ ampicillin was supplemented for plasmid propagation. S. cerevisiae strains were grown in YP medium $(20 \mathrm{~g} / \mathrm{l}$ tryptone and $10 \mathrm{~g} / \mathrm{l}$ yeast extract) supplemented with glucose (YPD), xylose (YPX), or glucose and xylose (YPDX) at $30^{\circ} \mathrm{C}, 250 \mathrm{rpm}$ and when necessary, antibiotics were added as follows: G418, $200 \mu \mathrm{g} / \mathrm{ml}$; zeocin, $200 \mu \mathrm{g} / \mathrm{ml}$; hygromycin, $200 \mu \mathrm{g} / \mathrm{ml}$.

\section{Plasmid construction}

Plasmids were constructed by conventional cloning methods. pCpA1/G-XI was constructed to integrate a copy of Piromyces XYLA encoding xylose isomerase at 
Table 2 Performance of metabolically engineered S. cerevisiae strains in anaerobic batch cultivation

\begin{tabular}{|c|c|c|c|c|c|c|c|c|c|}
\hline Strain & Description $^{a}$ & Condition & Initial sugar, g/l & Final ethanol ${ }^{b, c}, g / l$ & $Y_{E}^{b, c}, g / g$ & $Y_{G}^{b, c}, g / g$ & $R_{S}^{b, c}, g / l / h$ & $R_{E}^{b, c}, g / l / h$ & Reference \\
\hline RWB218 & $\begin{array}{l}\text { piXYLA; XKS1; TAL1; TKL1; RKI1; RPE1; } \triangle G R E 3 ; \\
\text { adaptive evolution }\end{array}$ & $\begin{array}{l}\text { Defined medium; inoculum } \\
\text { size at } 1.1 \mathrm{~g} \text { DCW/l; fermentation } \\
\text { time at } 24 \mathrm{~h}\end{array}$ & 100 (glu.) 25 (xyl.) & 47.5 & 0.38 & 0.08 & 5.21 & 1.98 & {$[4]$} \\
\hline H131-A3-AL ${ }^{C S}$ & $\begin{array}{l}\text { piXYLA; psXYL3; psTAL1; TKL1; RPE1; RKI1; } \\
\text { ARG4; LEU2; adaptive evolution }\end{array}$ & $\begin{array}{l}\text { Defined medium; inoculum size } \\
\text { at } 0.05 \mathrm{~g} \mathrm{DCW/l} \text {; fermentation } \\
\text { time at ca. } 30 \mathrm{~h}\end{array}$ & $40(x y l)$. & 16.4 & 0.41 & N/A & 1.33 & 0.55 & {$[8]$} \\
\hline GS1.11-26 & $\begin{array}{l}\text { CPXYIA; XKS1; TAL1; TKL1; RPE1; RKI1; HXT7; } \\
\text { TKL2; TAL2; pSARAT, blaraB; ecaraA; ecaraD; } \\
\text { adaptive evolution }\end{array}$ & 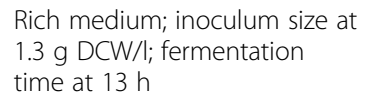 & 36 (glu.) 37 (xyl.) & 33.6 & 0.46 & $\mathrm{~N} / \mathrm{A}$ & 5.62 & 2.58 & [18] \\
\hline \multirow[t]{2}{*}{ CIBTS0735 } & $\begin{array}{l}\text { piXYLA; XKS1; TAL1; TKL1; RKI1; RPE1; CiGXF1; } \\
\text { adaptive evolution }\end{array}$ & $\begin{array}{l}\text { Rich medium; inoculum size at } \\
0.63 \mathrm{~g} \mathrm{DCW/l} \text {; fermentation } \\
\text { time at } 16 \mathrm{~h}\end{array}$ & 40 (xyl.) & 17.47 & 0.44 & 0.04 & 2.50 & 1.09 & This study \\
\hline & & $\begin{array}{l}\text { Rich medium; inoculum size at } \\
0.63 \mathrm{~g} \mathrm{DCW} / \text {; fermentation } \\
\text { time at } 24 \mathrm{~h}\end{array}$ & 80 (glu.) 40 (xyl.) & 53.34 & 0.44 & 0.04 & 5.00 & 2.22 & This study \\
\hline
\end{tabular}

apiXYLA, Piromyces XYLA; psXYL3, Pichia stipitis XYL3; psTAL1, P. stipitis TAL1; cpxyIA, Clostridium phytofermentans xyIA; psARAT, P. stipitis ARAT; blaraB, Bacillus subtilis araB; ecaraA, Escherichia coli araA; ecaraD, E. coli araD; ciGXF1, Candida intermedia GXF1. ${ }^{b} Y_{\mathrm{E}}$, ethanol yield; $\mathrm{Y}_{\mathrm{G}}$, glycerol yield; $\mathrm{R}_{\mathrm{S}}$, sugar consumption rate; $\mathrm{R}_{\mathrm{E}}$, ethanol production rate. ${ }^{\mathrm{C}}$ Values for all strains were recalculated based on the whole fermentation process. 
Table 3 Strains and plasmids used in this study

\begin{tabular}{|c|c|c|}
\hline Strain/plasmid & Description & Reference $^{\mathrm{a}}$ \\
\hline CCTCC M94055 & An industrial ethanol-producing S. cerevisiae strain; MATa/a & CCTCC \\
\hline CIBTS0525 & $\begin{array}{l}\text { S. cerevisiae CCTCC M94055 derivative; arg 1::pCpA1/G-XI; ty1::pYIE2-Ty-XI; } \\
\text { 8:IoxP-zeo-loxP-ADH1p-XKS1-XKS1t-TPI1p-TAL1-TAL1t-PGK1p-RPE1-RPE1t-FBA1p-TKL1-TKL1t-PDC1p-RK11-RKI1t }\end{array}$ & This study \\
\hline ClBTS0555 & A single-colony isolate of CIBTS0525 after adaptive evolution & This study \\
\hline CIBTS0573 & CIBTS0525 derivative; gre3::pYIE2-GXF1 & This study \\
\hline CIBTS0735 & A single-colony isolate of CIBTS0573 after adaptive evolution & This study \\
\hline $\mathrm{pCpA1/G-XI}$ & Carrying TPI1p-XYLA-CYC1t, used to integrate a copy of Piromyces XYLA at the ARG1 locus & This study \\
\hline pYIE2-Ty-XI & Carrying TPl1p-XYLA-CYC1t, used to integrate a copy of Piromyces XYLA at the Ty1 locus & This study \\
\hline pSH47-hph & pSH47 encoding a hygromycin-resistant protein & This study \\
\hline pYIE2-GXF1 & Carrying TPI1p-GXF1-TPI1t, used to integrate Candida intermidia GXF1 at the GRE3 locus & This study \\
\hline
\end{tabular}

${ }^{a}$ CCTCC, China Center for Type Culture Collection.

the ARG1 locus and pYIE2-Ty-XI was constructed to integrate the second XYLA copy at the Ty1 locus. For both plasmids, TPI1p (promoter of TPI1) and CYC1t (terminator of $C Y C 1$ ) were used to drive $X Y L A$ expression. Genetic maps of these two plasmids are shown in Additional file 1: Figure S4. pSH47-hph was constructed to rescue loxP-flanked selection markers by cloning a hygromycin-resistant gene into pSH47 [31]. pYIE2-GXF1 was constructed to integrate Candida intermedia GXF1 that encodes a xylose transporter at the GRE3 locus. For GXF1, TPI1p and TPI1t (terminator of TPI1) were used to drive gene expression. The genetic map of PYIE2-GXF1 is shown in Additional file 1: Figure S5.

\section{Strain construction}

All S. cerevisiae strains were constructed from S. cerevisiae CCTCC M94055. The process of strain construction was depicted in Figure 1. The first XYLA copy was integrated at the ARG1 locus by transforming Kpn2I-linearized pCpA1/G-XI using G418 as the selection marker and similarly, the second $X Y L A$ copy was integrated at the $T y 1$ locus by transforming SalI-linearized pYIE2-Ty-XI using zeocin as the selection marker. Then, zeocin rescue was performed as previously described using pSH47-hph [31]. Subsequently, a DNA fragment (Additional file 1: Figure S6) containing loxP-zeo-loxP, ADH1p-XKS1-XKS1t, TPI1p-TAL1-TAL1t, PGK1p-RPE1-RPE1t, FBA1p-TKL1TKL1t, and PDC1p-RKI1-RKI1t was directly assembled and inserted at the $\delta$ locus using DNA Assembler [32] and zeocin as the selection marker. After zeocin rescue, pYIE2-GXF1 was linearized by PstI and then transformed into CIBTS0525 to integrate GXF1 at the GRE3 locus, resulting in CIBTS0573.

\section{Adaptive evolution by serial transfer}

For adaptive evolution, strains were grown aerobically in YP medium supplemented with 20-40 g/l xylose. When cultures entered into the stationary phase, new cultivations were made by transferring $10 \%(\mathrm{v} / \mathrm{v})$ of the cultures into fresh media. This procedure was repeated ca. 15 cycles until the xylose consumption rate did not increase any more. Finally, cultures were diluted and plated on YPX agar plates for single-colony isolation.

\section{Anaerobic fermentation in rich media}

Strains were first grown aerobically in $5 \mathrm{ml} \mathrm{YP} \mathrm{medium}$ supplemented with $20 \mathrm{~g} / \mathrm{l}$ glucose and $10 \mathrm{~g} / \mathrm{l}$ xylose in $20 \mathrm{ml}$ test tubes overnight at $30^{\circ} \mathrm{C}, 250 \mathrm{rpm}$. Then, cultures were used to inoculate $30 \mathrm{ml}$ fresh media in $250 \mathrm{ml}$ flasks. After aerobic growth, cells from the late stationary phase were harvested, washed twice with sterile water, and later used to inoculate $100 \mathrm{ml}$ YP medium supplemented with $40 \mathrm{~g} / \mathrm{l}$ xylose or $80 \mathrm{~g} / \mathrm{l}$ glucose and $40 \mathrm{~g} / \mathrm{l}$ xylose in $300 \mathrm{ml}$ bottles capped with rubber stoppers (syringe needles were inserted into rubber stoppers to release $\mathrm{CO}_{2}$ during fermentation) at an initial $\mathrm{OD}_{600}$ of $1.0(0.63 \mathrm{~g} \mathrm{DCW} / \mathrm{l})$. During fermentation, samples were taken at intervals for analysis of $\mathrm{OD}_{600}$ and metabolites.

\section{Analytical methods}

Cell densities $\left(\mathrm{OD}_{600}\right)$ were determined using Beckman Coulter DU 730 Spectrophotometer. For determination of cell dry weight, different volumes $(0.5 \mathrm{ml}-1.5 \mathrm{ml})$ of cell cultures were collected by centrifugation. Cells were washed once with sterile water, dried in a $105^{\circ} \mathrm{C}$ oven for $48 \mathrm{~h}$, and then weighed. One $\mathrm{OD}_{600}$ unit corresponded to $0.63 \mathrm{~g} \mathrm{DCW} / \mathrm{l}$. Ethanol was detected using Agilent 7890A GC with an Alltech EC-WAX column and a flame ionization detector. The column was eluted at $85^{\circ} \mathrm{C}$ with nitrogen. Glucose, xylose, glycerol, and xylitol were detected using Agilent 1200 HPLC with a Bio-Rad HPX$87 \mathrm{H}$ column and a refractive index detector. The column was eluted at $65^{\circ} \mathrm{C}$ with $5 \mathrm{mM}$ of sulfuric acid at a flow rate of $0.6 \mathrm{ml} / \mathrm{min}$.

\section{Enzyme assay}

Strains were grown anaerobically to the early stationary phase in YP medium supplemented with $20 \mathrm{~g} / \mathrm{l}$ glucose 
and $10 \mathrm{~g} / \mathrm{l}$ xylose. Cells were harvested by centrifugation at room temperature and washed twice with sterile water. After suspended in Tris- $\mathrm{HCl}$ buffer $(50 \mathrm{mM}$, pH7.5), cells were disrupted by sonication. Cell debris was removed by centrifugation and crude extracts were reserved for enzyme assays. The total protein concentration in cell extracts was determined using the Bradford assay with bovine serum albumin as the standard. The activity of xylose isomerase was measured in reaction mixtures containing $50 \mathrm{mM}$ phosphate buffer (pH6.8), $1 \mathrm{mM} \mathrm{MnCl}, 5 \mathrm{mM}$ xylose, and cell extracts. After incubation at $37^{\circ} \mathrm{C}$ for $20 \mathrm{~min}$, xylulose was quantified by the cysteine-carbazole-sulfuric acid method [33].

\section{Additional file}

\section{Additional file 1: Supplemental material. Figure S1, Anaerobic} fermentation of CIBTS0552 on xylose. (A), Construction process of CIBTS0552. (B), Xylose fermentation. The strain was cultured at $30^{\circ} \mathrm{C}$ with shaking in YP medium supplemented with $40 \mathrm{~g} / \mathrm{l}$ xylose in a $300 \mathrm{ml}$ shake flask containing $100 \mathrm{ml}$ medium. The shake flask was capped with a rubber stopper (a syringe needle was inserted into the rubber stopper to release $\mathrm{CO}_{2}$ during fermentation). The initial $\mathrm{OD}_{600}$ was set at 1.0 (0.63 g DCW/I). Figure S2, Anaerobic fermentation of CIBTS0734 on xylose. (A), Construction process of CIBTS0734. (B), Xylose fermentation. The strain was cultured at $30^{\circ} \mathrm{C}$ with shaking in YP medium supplemented with 40 $\mathrm{g} / \mathrm{l}$ xylose in a $300 \mathrm{ml}$ shake flask containing $100 \mathrm{ml}$ medium. The shake flask was capped with a rubber stopper (a syringe needle was inserted into the rubber stopper to release $\mathrm{CO}_{2}$ during fermentation). The initial

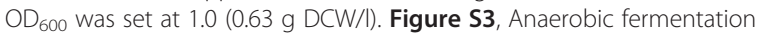
of CIBTS0735 in undetoxified corn stover hydrolysate. The strain was cultured at $30^{\circ} \mathrm{C}$ with shaking in a $100 \mathrm{ml}$ shake flask containing $30 \mathrm{ml}$ undetoxified corn stover hydrolysate. The hydrolysate was only supplemented with $1 \mathrm{~g} / \mathrm{l}$ urea and its pH was adjusted to 6.0. The shake flask was capped with a rubber stopper (a syringe needle was inserted into the rubber stopper to release $\mathrm{CO}_{2}$ during fermentation) and the initial $\mathrm{OD}_{600}$ was set at $8.0(5 \mathrm{~g}$ DCW/I). The hydrolysate contained $82.3 \mathrm{~g} / \mathrm{l}$ glucose, $54.2 \mathrm{~g} / \mathrm{l}$ xylose, $8.4 \mathrm{~g} / \mathrm{l}$ acetic acid and $2.6 \mathrm{~g} / \mathrm{HMF}$. Figure $\mathbf{S 4}$, Genetic maps for $\mathrm{pCpA} 1 / \mathrm{G}-\mathrm{XI}$ and pYIE2-Ty-XI. Figure S5, Genetic map for PYIE2-GXF1. Figure S6, DNA fragment used to overexpress xylulokinase and the four nonoxidative enzymes in the pentose phosphate pathway at the $\delta$ locus.

\section{Competing interests}

The authors declare that they have no competing interests.

\section{Authors' contributions}

$L D, J Y, Y J$ and SY designed the study, interpreted the results and wrote the manuscript. $Y L$ and FQ performed the experiments. All authors read and approved the final manuscript.

\section{Acknowledgements}

This work was supported by Novozymes A/S, Shanghai Postdoctoral Scientific Program (13R21417700), Postdoctoral Fellowship Program of Shanghai Institutes for Biological Sciences, Chinese Academy of Sciences (2013KIP307), National High-tech Research and Development Program of China (863: 2012AA02A704), National Basic Research Program of China (973: 2014CB745100), National Basic Research Program of China (973: 2012CB721105), National Key Technology R\&D Program (2012BAD32B07), and Knowledge Innovation Program of the Chinese Academy of Sciences (KSCX2-EW-J-12). The authors would like to thank Dr. Wenping Wu, Dr. Carsten Hjort, Dr. Guifang Wu, Dr. Wei Li, and Ms. Hongyi Yang at Novozymes A/S for valuable discussions. Thanks also go to Mr. Zhijun Li at Angel Yeast for sharing valuable information.
Received: 2 May 2013 Accepted: 10 December 2013

Published: 19 December 2013

\section{References}

1. Hahn-Hägerdal B, Karhumaa K, Fonseca C, Spencer-Martins I, Gorwa-Grauslund MF: Towards industrial pentose-fermenting yeast strains. App/ Microbiol Biotechnol 2007, 74:937-953.

2. Matsushika A, Inoue H, Kodaki T, Sawayama S: Ethanol production from xylose in engineered Saccharomyces cerevisiae strains: current state and perspectives. Appl Microbiol Biotechnol 2009, 84:37-53.

3. Kim SR, Ha SJ, Wei N, Oh EJ, Jin YS: Simultaneous co-fermentation of mixed sugars: a promising strategy for producing cellulosic ethanol. Trends Biotechnol 2012, 30:274-282.

4. Kuyper M, Toirkens MJ, Diderich JA, Winkler AA, van Dijken JP, Pronk JT: Evolutionary engineering of mixed-sugar utilization by a xylose-fermenting Saccharomyces cerevisiae strain. FEMS Yeast Res 2005, 5:925-934.

5. Kuyper M, Hartog MMP, Toirkens MJ, Almering MJH, Winkler AA, van Dijken JP, Pronk JT: Metabolic engineering of a xylose-isomerase-expressing Saccharomyces cerevisiae strain for rapid anaerobic xylose fermentation. FEMS Yeast Res 2005, 5:399-409.

6. Runquist D, Hahn-Hagerdal B, Rådström P: Comparison of heterologous xylose transporters in recombinant Saccharomyces cerevisiae. Biotechnol Biofuels 2010, 3:5.

7. Sedlak M, Ho NWY: Production of ethanol from cellulosic biomass hydrolysates using genetically engineered Saccharomyces yeast capable of cofermenting glucose and xylose. Appl Biochem Biotechnol 2004, 113-16:403-416.

8. Zhou H, Cheng JS, Wang BL, Fink GR, Stephanopoulos G: Xylose isomerase overexpression along with engineering of the pentose phosphate pathway and evolutionary engineering enable rapid xylose utilization and ethanol production by Saccharomyces cerevisiae. Metab Eng 2012, 14:611-622.

9. Hamacher T, Becker J, Gárdonyi M, Hahn-Hägerdal B, Boles E: Characterization of the xylose-transporting properties of yeast hexose transporters and their influence on xylose utilization. Microbiology 2002, 148:2783-2788.

10. van Maris AJ, Abbott DA, Bellissimi E, van den Brink J, Kuyper M, Luttik MA, Wisselink HW, Scheffers WA, van Dijken JP, Pronk JT: Alcoholic fermentation of carbon sources in biomass hydrolysates by Saccharomyces cerevisiae: current status. Antonie Van Leeuwenhoek 2006, 90:391-418.

11. Runquist D, Fonseca C, Rådström P, Spencer-Martins I, Hahn-Hägerdal B: Expression of the Gxf1 transporter from Candida intermedia improves fermentation performance in recombinant xylose-utilizing Saccharomyces cerevisiae. Appl Microbiol Biotechnol 2009, 82:123-130.

12. Johansson B, Hahn-Hägerdal B: The non-oxidative pentose phosphate pathway controls the fermentation rate of xylulose but not of xylose in Saccharomyces cerevisiae TMB3001. FEMS Yeast Res 2002, 2:277-282.

13. Träff $\mathrm{KL}$, Cordero RRO, van Zyl WH, Hahn-Hägerdal B: Deletion of the GRE3 aldose reductase gene and its influence on xylose metabolism in recombinant strains of Saccharomyces cerevisiae expressing the $x y I A$ and XKS1 genes. Appl Environ Microbiol 2001, 67:5668-5674.

14. Shen Y, Chen X, Peng B, Chen L, Hou J, Bao X: An efficient xylose-fermenting recombinant Saccharomyces cerevisiae strain obtained through adaptive evolution and its global transcription profile. App/ Microbiol Biotechno/ 2012 96:1079-1091.

15. Meinander NQ, Hahn-Hägerdal B: Fed-batch xylitol production with two recombinant Saccharomyces cerevisiae strains expressing XYL1 at different levels, using glucose as a cosubstrate: a comparison of production parameters and strain stability. Biotechnol Bioeng 1997, 54:391-399.

16. Zhang Z, Moo-Young M, Chisti Y: Plasmid stability in recombinant Saccharomyces cerevisiae. Biotechnol Adv 1996, 14:401-435.

17. Tanino T, Hotta A, Ito T, Ishii J, Yamada R, Hasunuma T, Ogino C, Ohmura N, Ohshima T, Kondo A: Construction of a xylose-metabolizing yeast by genome integration of xylose isomerase gene and investigation of the effect of xylitol on fermentation. App/ Microbiol Biotechnol 2010, $88: 1215-1221$

18. Demeke MM, Dietz H, Li Y, Foulquié-Moreno MR, Mutturi S, Deprez S, Den Abt T, Bonini BM, Liden G, Dumortier F, et al: Development of a D-xylose fermenting and inhibitor tolerant industrial Saccharomyces cerevisiae strain with high performance in lignocellulose hydrolysates using metabolic and evolutionary engineering. Biotechnol Biofuels 2013, 6:89. 
19. Huang H, Guo X, Li D, Liu M, Wu J, Ren H: Identification of crucial yeast inhibitors in bio-ethanol and improvement of fermentation at high $\mathrm{pH}$ and high total solids. Bioresour Technol 2011, 102:7486-7493.

20. Kang D, Zhao L: Comparison of the fermentation performance of several strains of achohol yeast. Liquor-making Sci Technol 2006, 140:40-43.

21. Peng B, Shen Y, Li X, Chen X, Hou J, Bao X: Improvement of xylose fermentation in respiratory-deficient xylose-fermenting Saccharomyces cerevisiae. Metab Eng 2012, 14:9-18

22. Parachin NS, Bergdahl B, van Niel EW, Gorwa-Grauslund MF: Kinetic modelling reveals current limitations in the production of ethanol from xylose by recombinant Saccharomyces cerevisiae. Metab Eng 2011, 13:508-517.

23. Tanino T, Ito T, Ogino C, Ohmura N, Ohshima T, Kondo A: Sugar consumption and ethanol fermentation by transporter-overexpressed xylose-metabolizing Saccharomyces cerevisiae harboring a xyloseisomerase pathway. J Biosci Bioeng 2012, 114:209-211.

24. Fujitomi K, Sanda T, Hasunuma T, Kondo A: Deletion of the PHO13 gene in Saccharomyces cerevisiae improves ethanol production from lignocellulosic hydrolysate in the presence of acetic and formic acids, and furfural. Bioresour Technol 2012, 111:161-166.

25. Hasunuma T, Sung KM, Sanda T, Yoshimura K, Matsuda F, Kondo A: Efficient fermentation of xylose to ethanol at high formic acid concentrations by metabolically engineered Saccharomyces cerevisiae. Appl Microbiol Biotechnol 2011, 90:997-1004.

26. Koppram R, Albers E, Olsson L: Evolutionary engineering strategies to enhance tolerance of xylose utilizing recombinant yeast to inhibitors derived from spruce biomass. Biotechnol Biofuels 2012, 5:32.

27. Liu ZL: Molecular mechanisms of yeast tolerance and in situ detoxification of lignocellulose hydrolysates. App/ Microbiol Biotechnol 2011, 90:809-825.

28. Wright J, Bellissimi E, de Hulster E, Wagner A, Pronk JT, van Maris AJ: Batch and continuous culture-based selection strategies for acetic acid tolerance in xylose-fermenting Saccharomyces cerevisiae. FEMS Yeast Res 2011, 11:299-306.

29. Sauer U: Evolutionary engineering of industrially important microbial phenotypes. Adv Biochem Eng Biotechnol 2001, 73:129-169.

30. Kuyper M, Winkler AA, van Dijken JP, Pronk JT: Minimal metabolic engineering of Saccharomyces cerevisiae for efficient anaerobic xylose fermentation: a proof of principle. FEMS Yeast Res 2004, 4:655-664.

31. Güldener U, Heck S, Fiedler T, Beinhauer J, Hegemann JH: A new efficient gene disruption cassette for repeated use in budding yeast. Nucleic Acids Res 1996, 24:2519-2524.

32. Shao Z, Zhao H: DNA assembler, an in vivo genetic method for rapid construction of biochemical pathways. Nucleic Acids Res 2009, 37:e16

33. Dische Z, Borenfreund $\mathrm{E}: \mathrm{A}$ new spectrophotometric method for the detection and determination of keto sugars and trioses. J Biol Chem 1951, 192:583-587.

doi:10.1186/1472-6750-13-110

Cite this article as: Diao et al:: Construction of fast xylose-fermenting yeast based on industrial ethanol-producing diploid Saccharomyces cerevisiae by rational design and adaptive evolution. BMC Biotechnology $201313: 110$

\section{Submit your next manuscript to BioMed Central and take full advantage of:}

- Convenient online submission

- Thorough peer review

- No space constraints or color figure charges

- Immediate publication on acceptance

- Inclusion in PubMed, CAS, Scopus and Google Scholar

- Research which is freely available for redistribution 